\title{
Subject-Based Modular Eigenspace Scheme for Face Recognition
}

\author{
Bai-ling Zhang ${ }^{1}$, Min-yue Fu ${ }^{1}$ and Hong Yan $^{2}$ \\ 1 Department of Electrical and Computer Engineering, \\ University of Newcastle, NSW 2308, Australia \\ 2 Department of Electrical Engineering, \\ University of Sydney, NSW, 2006, Australia
}

\begin{abstract}
Face recognition is an important research area with many potential applications such as biometric security. Among various techniques, eigenface method by principal component analysis (PCA) of face images has been widely used. In traditional eigenface methods, PCA was used to get the eigenvectors of the covariance matrix of a training set of face images and recognition was achieved by applying a template matching scheme with the vectors obtained by projecting new faces along a small number of eigenfaces. In order to avoid the time consuming step of recomputing eigenfaces when new faces are added, we use a set of modules to generate PCA based face representation for each subjects instead of PCA of entire face images. The localized nature of the representation makes the system easy to maintain and tolerant of local facial characteristic changes. Results indicate that the modular scheme yield accurate recognition on the widely used Olivetti Research Laboratory (ORL) face database.
\end{abstract}

\section{Introduction}

In recent years considerable effort has been made toward machine recognition of human face images and the need for accurate and robust performance is increasing and alhough much progress has been made, it remains a difficult problem. As there are many variabilities of face images such as illumination condition, background, orientation, view, hair, glasses, and expression, a successful face recognition system has to be robust with respect to the variabilities and capture the essential similarities for any given human face.

A face image is usually treated as a vector of pixel intensities which belong to a very high dimensional space. This gives rise to the well-known difficulty called the curse of dimensionality which says that there is hardly ever enough data to robustly train a classifier in high dimensional space [1]. One way to overcome the difficulty is to construct efficient low dimensional representations which are sufficient for the classification task. The principal component analysis is such an approach, which explicitly represent the faces as a weighted sum of

\footnotetext{
* email: bailing@ee.usyd.edu.au
} 
eigenvectors (called eigenfaces) extracted from the covariance matrix of a set of face images [3-4,7-8]. Eigenfaces best account for the distribution of face images within the entire image space and yields statistically uncorrelated coefficients which are optimal in the mean-square-error sense for reconstruction. PCA type representation avoids the difficult problem of feature selection and extraction in geometrical template methods. It is generally recognized that using eigenfaces is a practical technique which is fast and simple and produces good performance.

In previous eigenface-based methods, the main problems are the need for representation at a range of scales and orientations which causes extra complexity, and updating the average eigenfaces when new faces are added to the data set. On the other hand, the system size and training times for such a standard global eigenface model will become impractical as the number of classes increases. In the perceptual framework for human face processing proposed by Hay and Young [9], a concept of face recognition unit was suggested in which each unit produces a positive signal only for the particular person it is trained to recognize. Based on this idea, we propose an improved eigenfaces scheme to allow fast small modules trained with examples of views of the person to be recognized. These face modules should give high performance and also alleviate the problem of adding new data to an existing trained system, which would otherwise have to be retrained. By spliting the training for individual classes into separate modules, our modular structure can potentially support large numbers of classes.

\section{ORL face database and preprocessing}

We test our recognition program on the database of face images produced by the Olivetti Research Laboratory (ORL) in Cambridge, U.K. The ORL database has become a benchmark as it has been widely used by many researchers. The database contained 10 face images of each of 40 persons (subjects). For some of the subjects, the images were taken at different times. There are considerable variations in the facial expression, angle to the camera and some variations in scale of up to about $10 \%$. For the subjects who wear glasses, the face images contain both cases with and without glasses. The images are greyscale with a resolution of $92 \times 112$. For computational convenience, we compress the faces by local averaging, using a $5 \times 5$ window giving $19 \times 23=437$ pixel images. This simple dimensionality reduction can be considered as a preliminary stage for extracting low-dimensional features from the original representational space. In Figure 1, an original face and its compressed one were illustrated. Reducing the number of pixels in an image filters out part of the detailed information, preserving only the low spatial frequency information. This problem is not very serious, however, since it has been shown [10] that there is enough information in a $32 \times 32$ pixel image of a digitized face with a resolution of 8 gray levels, for faces to be correctly identified by human subjects.

In our experiment, the set of images was partitioned into training and test 
sets, with 5 images of each person used for training and the remaining 5 for testing. A different eigenface module which was trained by the respective face images was set up for each person.
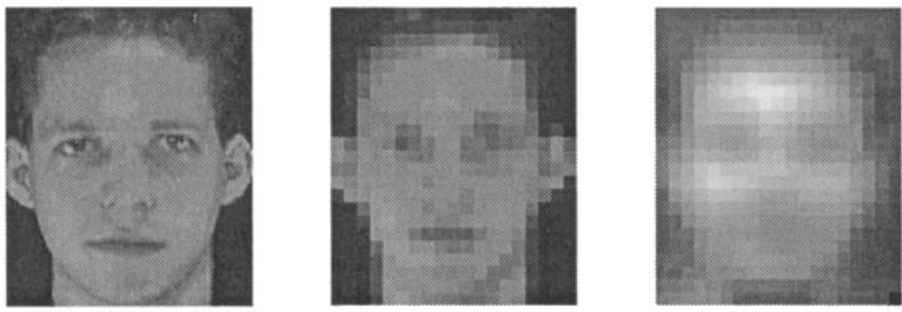

Fig. 1. A face image of subject No.1 (left), its compressed form by local average using a $5 \times 5$ window (middle) and the average face over all the $5 \times 40$ compressed facial images (right).

\section{Subject-based eigenface recognition scheme}

The face recognition problem can be simply stated as: Given a set of face images labelled with the person's identity (the learning set) and an unlabelled set of face images from the same group of people (the test set), identify the name of each person in the test images. With $N$ individuals under $M$ different views, one can do recognition in a universal eigenspace computed from the combination of $N \times M$ images. Our alternative formulation is to build a subject-based set of $N$ separate eigenspaces, each capturing the variations of the respective subject. Such a modular classification scheme has been successfully applied to handwritten digit recognition and signature verification in our previous studies. The subject-based eigenspace can also be considered as an extension to multiple sets of eigenvectors of the eigenface technique.

We establish a modular classification scheme for face recognition based on the traditional eigenface method. In our scheme, each subject has an independent eigenface space. For a specific $k$ th person, let the set of training images be $\mathbf{t}_{1}^{(\mathrm{k})}, \mathbf{t}_{2}^{(\mathrm{k})}, \cdots, \mathbf{t}_{\mathrm{M}}^{(\mathrm{k})}, k=1, \cdots, K$, where $M$ is the number of training images for the $k$ th person and $K$ the number of subjects. Each image is represented by a vector of length $L$, where $L$ is the number of pixels in the image. The average face is defined as $\mathbf{a}=\frac{1}{\mathrm{MK}} \sum_{\mathrm{k}=1}^{\mathrm{K}} \sum_{\mathrm{m}=1}^{\mathrm{M}} \mathbf{t}_{\mathrm{m}}^{(\mathrm{k})}$. The average face image is illustrated in Figure 1(c). The mean-centered set of vectors $x_{i}$ is then obtained by subtracting each input image from the average face, $x_{i}^{(k)}=t_{i}^{(k)}-a$. This scheme seeks to use PCA to find a set of $N$ orthogonal vectors $\mathbf{u}_{\mathrm{n}}$, which best describe the 
distribution of the data. The covariance matrix for the mean-centered vectors is:

$$
v^{(k)}=\frac{1}{M} \sum_{m=1}^{M} \mathbf{x}_{\mathrm{m}}^{(\mathrm{k})} \mathbf{x}_{\mathrm{m}}^{(\mathrm{k}) \prime}, \mathrm{k}=1, \cdots, \mathrm{K}
$$

Altogether there are $l \leq L^{2}$ eigenvectors $\mathbf{u}_{(l)}^{(\mathrm{k})}$ which can perfectly represent the input image set. Due to the statistics of face images, only a small number $n$ of eigenvectors $\left(n \ll L^{2}\right)$ can account for almost all of the variance in the input data. Therefore, if we use only the first $n$ dominant eigenvectors as orthogonal axes for projecting new face images, we achieve considerable computational saving. In the pattern recognition literature, this is the basis of the subspace method, for which many neural network learning algorithms are available. In this paper we directly perform singular value decomposition (SVD) of $v^{(k)}$ and apply the subspace spanned by those dominant eigenvectors (eigenfaces) of $\mathrm{v}^{(\mathrm{k})}$ to represent the subject. In Figure 2, we illustrate the ghostly "eigenfaces" for subject No.1. These eigenvectors can be thought of as a set of "global features" or "macrofeatures" from which the face is built.

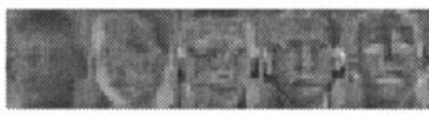

Fig. 2. First five eigenfaces for the No.1 subject in the face database as shown in Fig. 1.

Turk and Pentland [3] used eigenfaces for face identification via the following routine. When the eigenfaces were synthesized, every face in the database was represented as a vector of weight obtained by projecting an image onto the eigenface components through the simple inner product operation. When a test image was presented for identification, the new image was also represented by its vector of weights and the identification was done by retrieving the image in the database whose weights were the closest to the weights of the test image in Euclidean distance. In this paper, we realize verification based on the best reconstruction property of PCA. Specifically, a testing face $\mathrm{x}$ can be represented by a module $k$ in the system as a weighted sum of eigenvectors

$$
\begin{aligned}
\hat{\mathbf{x}}^{k} & =\sum_{l=1}^{N} \mathbf{u}_{l}^{\mathrm{k}} \mathbf{u}_{l}^{\mathrm{k}^{\prime}} \mathbf{x} \\
& =\sum_{l=1}^{N} \gamma_{l} \mathbf{u}_{l}^{\mathrm{k}} \mathrm{k}=1, \cdots, \mathrm{K}
\end{aligned}
$$

where $\gamma_{l}=\mathbf{u}_{l}^{\mathrm{k}^{\prime}} \mathbf{x}$ are the projections of face $\mathbf{x}$ onto the eigenvectors $\mathbf{u}_{l}^{\mathrm{k}}$. These coefficients can be interpreted as an indication of the extent to which a given eigenvector characterizes a particular face. 


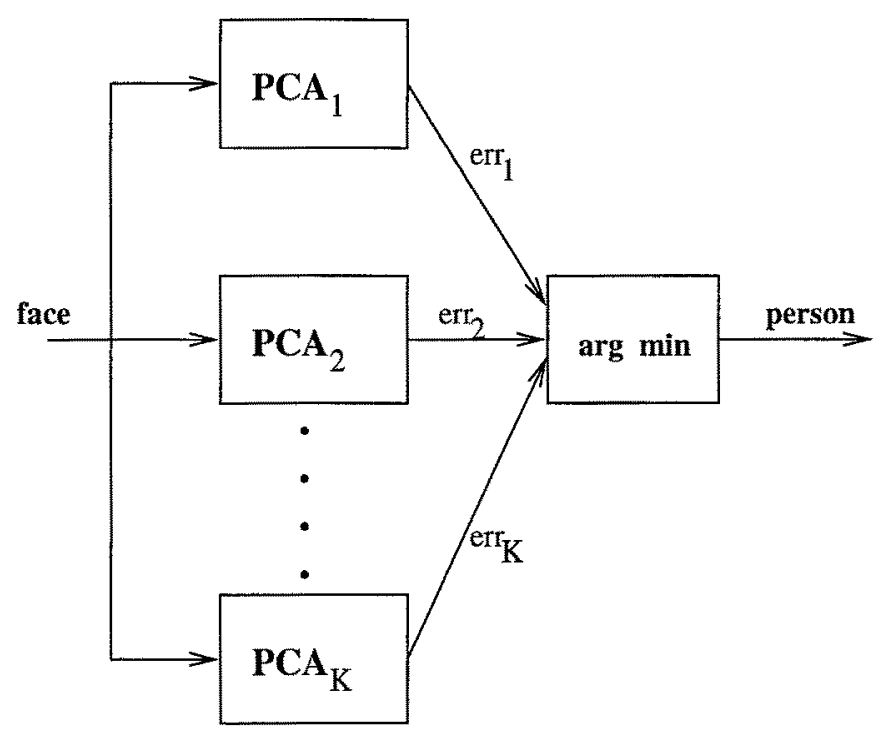

Fig. 3. The modular recognition scheme. Each module is a subject-based eigenfaces model.

From our modular eigenface recognition scheme illustrated in Figure 3, we calculate reconstructions from all the modules for a given testing face according to eqn (2) and record the respective reconstruction errors

$$
e r r_{k}=\left\|\mathbf{x}-\hat{\mathbf{x}}^{\mathbf{k}}\right\|^{2}, \mathbf{k}=1, \cdots, \mathbf{K}
$$

where $k$ indicates the number of the module in the system. A decision is then made by comparing these reconstruction errors to assign the face $\mathbf{x}$ to the $k^{*}$ th person if

$$
k^{*}=\arg \min _{k} e r r_{k}
$$

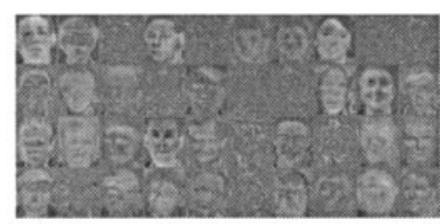

Fig. 4. 40 parallelly computed, reconstructed faces when a testing face from subject 1 is presented to the modular system.

The process of identifying a face is demonstrated further in Figure 4 when a testing face of subject No.1 is presented to the modular recognition system. 
The 40 modules simultaneously calculate reconstructions as illustrated in Figure 4 and the comparison module decides the first subject according to the reconstruction errors as shown in Figure 5.

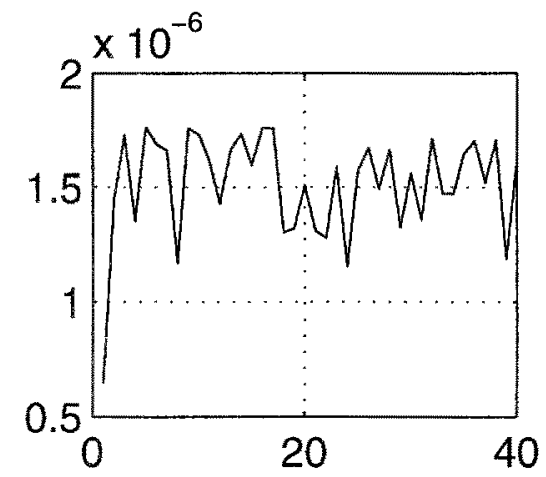

Fig. 5. Normalized reconstruction errors corresponding to the reconstructions in Fig. 4.

Table 1. Recognition result on the test face images. The number in the "recog." column stands for the number of faces successfully recognized among the five test face images for each subject.

\begin{tabular}{|c|c||c|c||c|c|c|c|}
\hline Subj. No. & Recog. & Subj. No. & Recog. & Subj. No. & Recog. & Subj. No. & Recog. \\
\hline 1 & 5 & 11 & 5 & 21 & 5 & 31 & 5 \\
\hline 2 & 5 & 12 & 5 & 22 & 5 & 32 & 5 \\
\hline 3 & 5 & 13 & 5 & 23 & 5 & 33 & 5 \\
\hline 4 & 5 & 14 & 5 & 24 & 5 & 34 & 5 \\
\hline 5 & 4 & 15 & 5 & 25 & 5 & 35 & 5 \\
\hline 6 & 5 & 16 & 4 & 26 & 4 & 36 & 5 \\
\hline 7 & 5 & 17 & 2 & 27 & 5 & 37 & 5 \\
\hline 8 & 5 & 18 & 5 & 28 & 4 & 38 & 5 \\
\hline 9 & 4 & 19 & 5 & 29 & 5 & 39 & 5 \\
\hline 10 & 4 & 20 & 5 & 30 & 5 & 40 & 5 \\
\hline
\end{tabular}

Some previous work suggested that different ranges of eigenvectors represent different kinds of information in faces [7-8]. Eigenvectors with large eigenvalues seem to capture information that is common to subsets of faces. Eigenvectors with small eigenvalues tend to capture information specific to individual faces. For most faces in the ORL database, we have found that 5-6 eigenvectors in a module is sufficient for identification. In the experiment, the first 5 of 10 images available for each subject were used for training and the remaining 5 
used for testing. For some faces such as those from subject No.17 which have much more variation in illumination, view, expression, etc., an increased number of eigenvectors will improve the recognition accuracy. In Table 1 , we give another experimental result by assigning 6 eigenvectors to most modules except modules No.3, No.5, No.9, No.10, No.14, No.16, No.17, No.26, No.28, No.32 and No.36 which were assigned 20 eigenvectors. As in the previous experiment, the five testing faces in each subject were in turn presented to the recognition system and we recorded the number of faces recognized successfully. In this experiment, the recognition rate was $96 \%$.

\section{Discussion and Conclusion}

The task of recognizing faces is inherently a classification problem in high dimensional feature space and thus subject to the "curse of dimensionality". The dimensionality-reducing properties of PCA have been applied to represent human faces in the eigenface method. By PCA, data are projected onto the leading eigenvectors of their covariance matrix, corresponding to the directions of maximum variance. Eigenfaces achieve recognition by representing both stored images and each new input as linear combinations of the estimated principal components (eigenfaces). In this paper, we propose a variation of this popular technique called subject-based modular eigenfaces. For each individual, a separate eigenface module is trained to discriminate between that person and others. The modular architecture allows us to add more data and update the recognition system.

Linear reconstruction of the original data from principal component projections is optimal in the mean square error sense. However, PCA is not optimal for classification and its limitations have been much discussed. In recent years, the PCA technique has been developed in several directions, for example, principal curve analysis, independent component analysis, etc. Among these developments, a simple mixture model of local PCA appears attractive. This model partitions a data set into a number of nonoverlapping regions with each region represented by a localized subspace. Obviously, our modular eigenface recognition system can be considered as a mixture of local PCA, which again demonstrate its advantages.

\section{References}

1. Intrator, N., Reisfeld, D., and Yeshurun, Y.: Face recognition using hybrid supervised/unsupervised neural network. Pattern Recognition Letters 19 (1996) 67-76

2. Oja, E.: Subspace methods of pattern recognition. Research Studies Press, Letchworth, U.K. 1983.

3. Turk, M., Pentland, A.: Eigenfaces for recognition. Journal of Cognitive Neuroscience 3(1) (1991) 70-86

4. Pentland, A., Moghaddam, B., and Starner, T.: View-based and modular eigenspaces for face recognition. IEE Proc.-Vis. Image Signal Process. 144 (1997) $73-80$ 
5. Lawrence, S., Giles, C. L., Tsoi, A.C. and Back, A.D.: Face recognition: a convolutional neural-network approach. IEEE Trans. Neural Networks 8 (1997) 98 113

6. Lin, S.H., Kung, S.Y. and Lin, L.J.: Face recognition/detection by probabilistic decision-based neural network. IEEE Trans. Neural Networks 8 (1997) $114-132$

7. Valentin, D., Abdi, H., O'Toole, A.J., and Cottrell, G.W.: Connectionist models of face processing: A survey. Pattern recognition 27(9) (1994) $1209-1230$

8. Hay, D.C., Young, A., and Ellis, A.W.: Routes through the face recognition system. Quarterly Journal of Experimental Psychology: Human Experimental Psychology 43 (1991) 761-791

9. Samal, A., Iyengar,P.A.: Automatic recognition and analysis of human faces and facial expressions. Pattern Recognition 25 (1992) 65-77 